\title{
Seeing SPIOs Directly In Vivo with Magnetic Particle Imaging
}

\author{
Bo Zheng $\odot,{ }^{1}$ Elaine Yu, ${ }^{1}$ Ryan Orendorff, ${ }^{1}$ Kuan Lu, ${ }^{2}$ Justin J Konkle, ${ }^{3}$ Zhi Wei Tay, ${ }^{1}$ \\ Daniel Hensley, ${ }^{1,3}$ Xinyi Y Zhou, ${ }^{1}$ Prashant Chandrasekharan, ${ }^{1}$ Emine U Saritas, ${ }^{4,5}$ \\ Patrick W Goodwill, ${ }^{3}$ John D Hazle, ${ }^{6}$ Steven M Conolly ${ }^{1,7}$ \\ ${ }^{1}$ Department of Bioengineering, UC Berkeley, Berkeley, CA, USA \\ ${ }^{2}$ Triple Ring Technologies, Newark, CA, USA \\ ${ }^{3}$ Magnetic Insight, Alameda, CA, USA \\ ${ }^{4}$ Department of Electrical and Electronics Engineering, Bilkent University, Ankara, Turkey \\ ${ }^{5}$ National Magnetic Resonance Research Center (UMRAM), Bilkent University, Ankara, Turkey \\ ${ }^{6}$ Department of Imaging Physics, The University of Texas MD Anderson Cancer Center, Houston, TX, USA \\ ${ }^{7}$ Department of Electrical Engineering and Computer Science, UC Berkeley, Berkeley, CA, USA
}

\begin{abstract}
Magnetic particle imaging (MPI) is a new molecular imaging technique that directly images superparamagnetic tracers with high image contrast and sensitivity approaching nuclear medicine techniques-but without ionizing radiation. Since its inception, the MPI research field has quickly progressed in imaging theory, hardware, tracer design, and biomedical applications. Here, we describe the history and field of MPI, outline pressing challenges to MPI technology and clinical translation, highlight unique applications in MPI, and describe the role of the WMIS MPI Interest Group in collaboratively advancing MPI as a molecular imaging technique. We invite interested investigators to join the MPI Interest Group and contribute new insights and innovations to the MPI field.
\end{abstract}

Key words: Magnetic particle imaging, Superparamagnetic iron oxide, Interest group

\section{Introduction}

Magnetic particle imaging (MPI) is an emerging biomedical imaging technique using completely new hardware that directly images superparamagnetic iron oxide (SPIO) tracers (Fig. 1a, b). The signal contrast in MPI is only generated by SPIOs introduced to the body; biological tissues neither generate nor attenuate MPI signals [4-6] (Fig. 1c-e). This high image contrast and contrast-to-noise ratio for SPIO tracers in MPI result in sensitive detection and positive contrast for imaging magnetic particles. These features of MPI make this modality comparable to nuclear medicine clinical techniques like positron emission tomography (PET)

Correspondence to: Bo Zheng; e-mail: bozheng@berkeley.edu and single-photon emission computed tomography (SPECT), but without the use of ionizing radiation. The nonradioactive SPIO tracers used in MPI are safe for injection into the body even at the significant doses required to treat iron deficiency (anemia) [7-10]. Detecting and staging diseases with molecular contrast can add to anatomical imaging modalities such as magnetic resonance imaging (MRI) or X-ray computed tomography (CT) [11]. Given that in vivo MPI is relatively new to the field of molecular imaging, and that there is a need to further develop the hardware, reagents, and software, we reasoned that an interest group for MPI as part of the World Molecular Imaging Society would be an excellent forum for exchanging information that would advance this emerging imaging modality. Here, we provide a description and history of MPI, outline some of the most pressing challenges, highlight unique applications, and discuss how the 

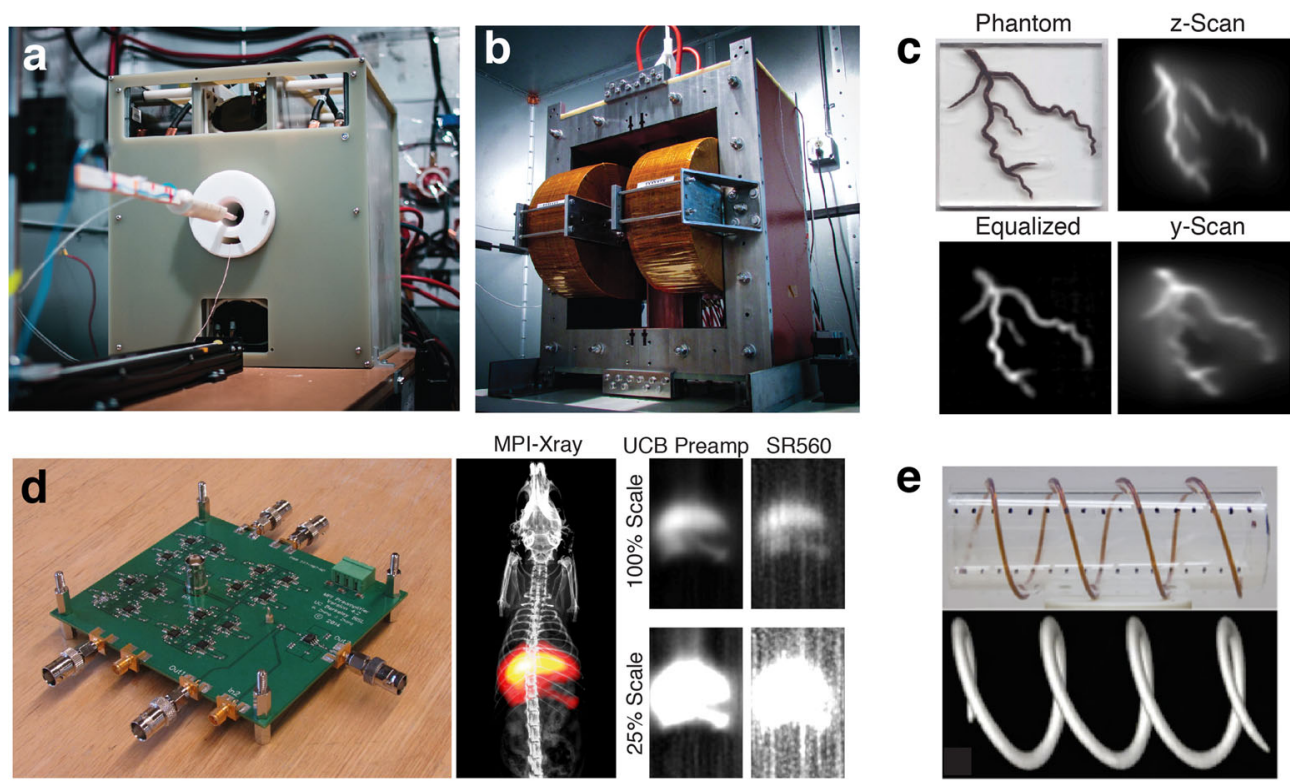

Fig. 1. Recent innovations in magnetic particle imaging. a Small-animal MPI scanners developed at UC Berkeley include a 7$\mathrm{T} / \mathrm{m}$ 3D field-free point (FFP) scanner and $\mathbf{b}$ a $6.5-\mathrm{T} / \mathrm{m}$ field-free line (FFL) scanner capable of producing 3D images via the filtered back-projection algorithm [1]. c Recent advances in MPI theory have also led to a significant reduction in image anisotropy and improvement in image conspicuity [2]. d Advances in detector electronics have enabled up to tenfold improvement in MPI detection sensitivity [3]. e The MPI signal is linear and quantitative with respect to the amount of SPIO tracer in the FOV, and MPI has image contrast comparable to PET and SPECT imaging.

MPI Interest Group will work to advance the field and fully integrate MPI into the molecular imaging arena.

MPI was invented and first demonstrated experimentally in 2005 by Philips Research in Hamburg, Germany [4]. Since then, there have been significant efforts toward bringing this new modality into preclinical and eventually clinical imaging environments. These include improving system hardware to increase resolution and sensitivity [1, 12-15]; developing the underlying system theory for MPI (akin to the development of $k$-space techniques and pulse sequences for MRI) [5, 16-20]; producing SPIO nanoparticles specifically for MPI to achieve higher resolution, greater sensitivity, and controlled circulation time [21, 22]; developing preclinical applications for MPI [6, 23-26]; and exploring safety and scalability for building human-sized MPI systems [27-30]. In 2010, the first International Workshop on Magnetic Particle Imaging was held in Lübeck, Germany with 70 attendees. This number has increased to over 200 recently and continues to grow each year. We feel that by highlighting some of the advances through MPI targeted sessions at the WMIC, we can build bridges between groups working on hardware, chemistry, biology, and medicine to work together to address those biological problems that are particularly well suited for MPI.

\section{How MPI Works}

The physics governing MPI is completely different from MRI, and it is not possible to perform MPI in an MRI scanner. The MPI signal is generated from SPIO tracers as they respond to applied magnetic fields. SPIOs, described by Langevin physics, magnetically align with applied magnetic fields until they reach superparamagnetic saturation. For spatial encoding of the MPI signal, we use a strong gradient field to magnetically saturate all SPIOs outside a central field-free region (FFR) [4]. Shifting this FFR through the imaging volume causes SPIOs in the FFR to rapidly and nonlinearly change magnetization, which produces a detectable voltage signal. This allows for straightforward image reconstruction where the instantaneous detector voltage signal is directly gridded to a 3D image space through knowledge of the FFR trajectory. Importantly, the induced signal is linearly proportional to the quantity of the SPIO tracer (Fig. 2e), enabling a linear and shift-invariant imaging method with a well-defined point spread function (PSF) [5, 18, 33].

The FFR used in MPI can be in the shape of a field-free point (FFP), which produces inherently 3D images, or a field-free line (FFL), which produces projection images akin to projection X-ray images. As in X-ray $\mathrm{CT}$, these projections can be used to make high-resolution 3D images using standard projection reconstruction algorithms. In MPI, the FFL approach can achieve up to orders of magnitude higher speed for projection imaging or SNR for projection reconstruction over FFP scanning [1, 19, 34].

\section{State of the Art}

There are currently more than seven groups in the world with operational custom-built MPI scanners [6, 15, 16, 35-37]. Two 


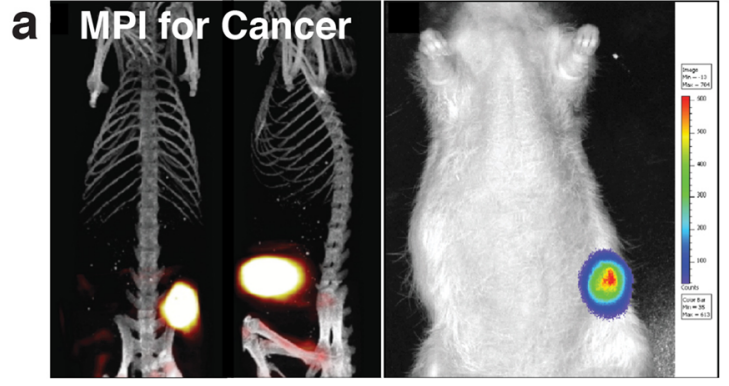

MPI 6 hours post-injection

Bioluminescent
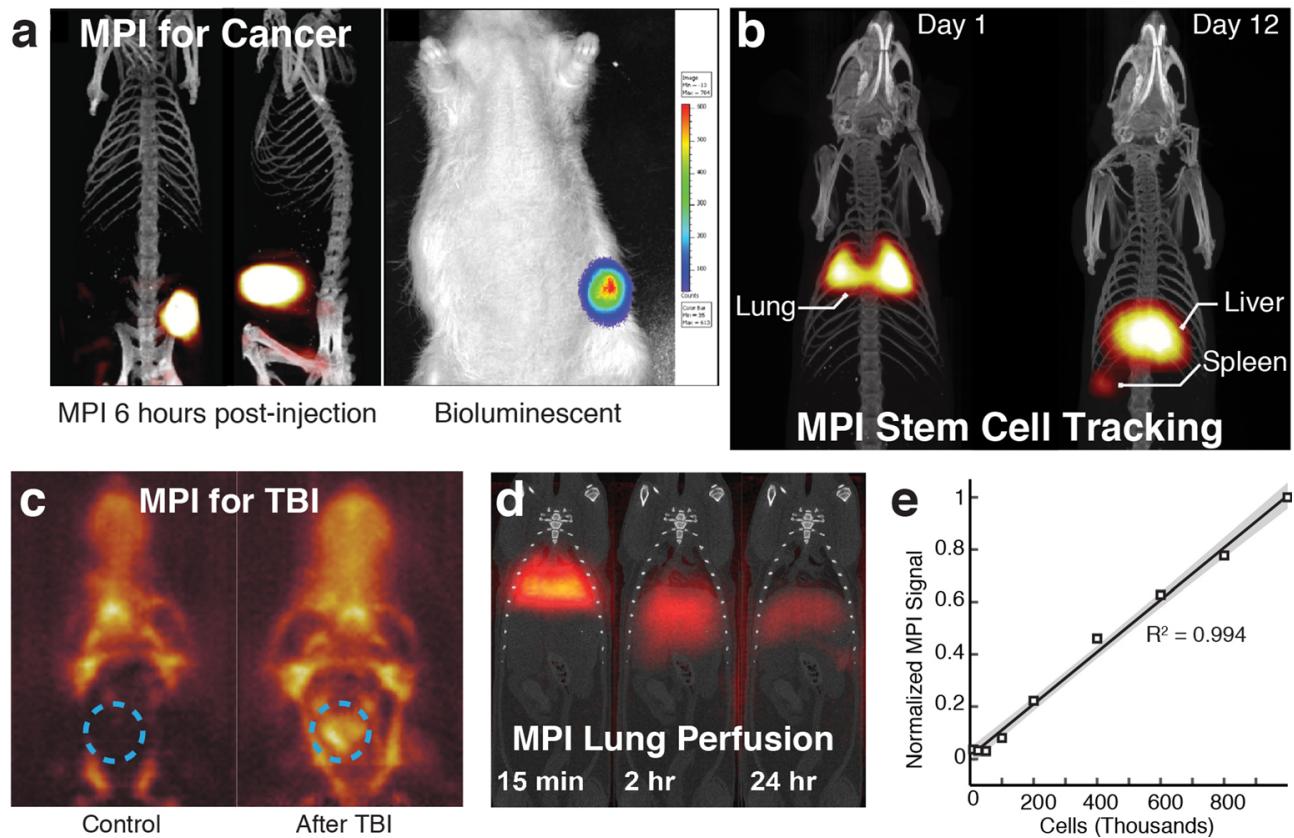

Fig. 2. Selected preclinical applications where magnetic particle imaging excels and can address unmet needs in biomedicine. a MPI/CT of a human breast tumor xenograft in rat shows enhanced image contrast $6 \mathrm{~h}$ after SPIO injection, compared to bioluminescent imaging of the tumor [31]. b MPI/CT images of intravenously injected stem cells trapped in the lungs (at day 1) and then cleared to the liver (at day 12) [25]. c Maximum-intensity projection MPI shows image contrast from hemorrhageinducing traumatic brain injury (right) compared to control (left) after SPIO administration. d MPI imaging of lung capillary perfusion using custom SPIO-macroprotein tracers. e The MPI signal is directly proportional to SPIO tracer in each voxel [32].

recent UC Berkeley MPI scanners are shown in Fig. 1a, b. As the MPI field is nascent and a standardized MPI system theory is yet to be adopted, each group uses differing and rapidly evolving scanner designs, pulse sequences, and reconstruction schemes. The dominant MPI scanning theories include the system matrix [4] and the $x$-space [18] approaches, which use different methods of image acquisition and reconstruction but are similar in their analysis of the fundamental MPI signalencoding mechanism [17, 33].

Most existing MPI systems are limited to small animalsized bores with free bores up to around $119 \mathrm{~mm}$ [38] and magnetic gradient strengths up to $7 \mathrm{~T} / \mathrm{m}$ [39]. Image acquisition times with these scanners have been shown to be as low as $20 \mathrm{~ms}$ per acquisition for smaller fields of view [38]. As a demonstration of the sensitivity of detection for labeled cells, our scanners have achieved as low as 200-cell $(5.4 \mathrm{ng})$ iron detection in a voxel [24]. This translates to roughly $130 \mathrm{nM}$ tracer concentration. Given this level of sensitivity, MPI may soon compete with nuclear medicine in "dose-limited sensitivity" for cell tracking. MPI sensitivity and contrast are already competitive with MRI cell tracking applications [40]. This sensitivity has not yet achieved the true physics limit, and further optimization of the detector coil and electronics could lead to the sensitivity potentially reaching a detection limit of picograms of iron per voxel with scan times of only seconds to minutes [38]. Figure 1a, b provides a sense of the geometry, size, and components of MPI scanners developed at UC Berkeley. The systems in academic labs are functional but lack the esthetics of commercial systems.

Commercial scanners have only recently become available from two companies: Bruker Biospin [41] and Magnetic Insight, Inc. [42]. Both of these systems are currently being evaluated in academic labs in the USA and Europe. Clinical translation of MPI systems is feasible but has not yet been achieved commercially, with several remaining challenges outlined below.

\section{Recent Challenges in MPI}

Being a relatively new imaging modality, there are several key areas in which improvements to MPI can have a significant impact. These include engineering in hardware, scanning, and image reconstruction, and here, we highlight three current and recent challenges:

1. Direct feedthrough interference: Unlike in MRI, where the detected nuclear precession signal is acquired microseconds to milliseconds after the RF excitation pulse, in MPI, the transmit and receive signals occur simultaneously. The applied drive field is typically an extremely pure tone, with ultra-low harmonic content. Any harmonics observed in the signal must be generated by the tracer. However, the receiver coil can also pick up a strong feedthrough interference at the fundamental frequency. Indeed, this 
direct feedthrough interference can be over six orders of magnitude larger than the signals from the SPIO tracer. To address this issue, all MPI scanners today remove the direct feedthrough interference via a strong bandstop filter [14, 43]. But this also removes the first harmonic of the SPIO signal spectrum, which hurts SNR and complicates image reconstruction. Fortunately, this can be recovered via a robust continuity algorithm, which preserves image linearity and shift-invariance [33]. Obviating the direct feedthrough filter would be an advance in MPI.

2. Isotropic resolution: The PSF in MPI is well defined analytically but is strongly asymmetric; that is, the PSF is wider in the axes perpendicular to the drive field scanning trajectory [18]. This results in directional blurring that makes image interpretation difficult (see Fig. 1c). We have recently shown that a simple combination of MPI signals acquired using orthogonal drive fields is sufficient to achieve isotropic resolution in MPI, and a subsequent equalization filter can de-emphasize low spatial frequencies and successfully remove background image haze [2].

3. Detector noise matching: Typical MPI scanners use detector coils with a signal bandwidth from $20 \mathrm{kHz}$ to $1 \mathrm{MHz}[14,38]$. In this bandwidth, it is difficult to achieve patient-noise dominance due to the relatively higher thermal noise of the detector system [44]. Moreover, it is difficult even to achieve wideband detector coil-noise dominance over the noise of the preamplifier due to the reactance of the detector coil. Thus, several signal amplification techniques, including transformer-coupling and parallel amplification, have been implemented to achieve exceptionally low amplifier noise for MPI (Fig. 1d) [3, 44].

\section{MPI Applications}

As a molecular and cellular imaging technique, MPI is well suited for preclinical and clinical applications that require high image contrast with little background tissue signal. One of the first applications of MPI described in the literature is for vascular and blood pool imaging $[16,38]$, where the high temporal resolution of MPI was used to visualize cardiac dynamics in real time [6]. Other applications of MPI toward vascular and blood pool imaging include angiography, pulmonary perfusion [32], and traumatic brain injury (see Fig. 2c, d). The ability to image dynamics without tissue background signal in MPI may also make it well suited for applications in assessing functional brain physiology using optimized long-circulating SPIO tracers [21, 45].

Another area of application in MPI is for tracking specific cell populations, such as cell-based therapies, as they redistribute and localize in vivo. Our group and others have recently shown the ability to track exogenously labeled human stem cells both in the murine brain and systemically for weeks to months $[24,25,46]$, with a detection sensitivity as low as 200 cells in a voxel. The capability to image quantitatively, sensitively, and longitudinally makes MPI uniquely well suited for cell tracking applications where monitoring the long-term localization and persistence of small cell populations is desired, both for preclinical research and in the clinic. Although MPI is still subject to the constraints of nanoparticle-based contrast, the fact that MPI images the particle directly and not its effect on water means that there are a greater range of particle configurations that could be developed with improved imaging characteristics. This chemical space has not been fully explored and is an area of development that would lead to a diverse range of unexplored imaging opportunities with multiplexing color-contrast capability.

Sensitive cancer diagnosis, staging, and treatment monitoring may also be facilitated by the availability of an ionizing-radiation-free imaging modality like MPI. Recently, we used MPI to image the vascular dynamics of a xenograft tumor model by exploiting the EPR effect (Fig. 2a, [31]). For future clinical applications, we anticipate the use of MPI in concert with biologically targeted approaches or immunotherapeutic approaches by, for example, labeling activated immune cells or immune checkpoint markers with SPIO tracers. Such approaches may allow for sensitive, real-time monitoring feedback on the efficacy of immunotherapies for both solid and diffuse tumors.

\section{WMIS MPI Interest Group Addresses the Emerging Challenges and Opportunities}

The rapidly developing MPI field is well poised for expansion to many more technical and preclinical molecular imaging research groups. The volume of literature in seminal MPI research already available, combined with the availability of commercial preclinical scanners from Magnetic Insight and Bruker, makes it feasible for other molecular imaging researchers to jump into the MPI field quickly.

There remain many unsolved challenges in MPI research, which today is similar in maturity to the state of MRI research in the early 1980s. For groups interested in developing MPI technology, challenges include (a) improving the spatial resolution of MPI, which is determined by SPIO tracer size and composition, applied magnetic fields, and SPIO relaxation times [47, 48]; (b) improving the detection sensitivity in MPI; (c) robust multi-color imaging [49, 50]; (d) theranostic MPI for guidance and real-time feedback on hyperthermia heating [51-53]; (e) improving tracer circulation time and image contrast [21, 45, 54]; (f) optimal combination of MPI with anatomic imaging modalities like CT or MRI for multi-modal imaging; (g) targeting SPIOs with great specificity to pathophysiology including cancer, cardiovascular disease, and stroke; and (h) scaling up preclinical MPI hardware to human MPI, while observing FDA and EU biosafety restrictions, including peripheral nerve stimulation (PNS) and tissue heating specific absorption rate (SAR) limits [27, 29]. These areas are the focus of the activities of the MPI interest group. 


\section{Conclusion}

In summary, there has been significant progress in the hardware, MPI-tailored SPIOs, and reconstruction algorithms for magnetic particle imaging over the past 10 years. Preclinical applications have really only begun within the past 5 years as commercial MPI scanners became available. The advent of commercial scanners presents new opportunities for WMIS investigators that were not previously possible. To this end, we invite interested molecular imaging researchers to the WMIS MPI Interest Group. We look forward to hearing about your contributions of new techniques and your valuable insights to the magnetic particle imaging field.

Acknowledgments. We are grateful for funding support from the Keck Foundation Grant 009323, NIH 1R01EB019458, NIH 1R24MH106053, and a UC Discovery Grant.

\section{Compliance with Ethical Standards}

\section{Conflict of Interest}

PWG and SMC are founders of Magnetic Insight. PWG, DWH, and JJK are employees of Magnetic Insight.

\section{References}

1. Konkle JJ, Goodwill PW, Carrasco-Zevallos OM, Conolly SM (2013) Projection reconstruction magnetic particle imaging. IEEE Trans Med Imaging 32:338-347

2. Lu K, Goodwill P, Zheng B, Conolly S (2015) Reshaping the 2D MPI PSF to be isotropic and sharp using vector acquisition and equalization. In: 2015 5th International Workshop on Magnetic Particle Imaging (IWMPI). IEEE, pp 1-1

3. Zhang W, Zheng B, Goodwill P, Conolly S (2015) A custom low-noise preamplifier for magnetic particle imaging. In: 2015 5th International Workshop on Magnetic Particle Imaging (IWMPI). pp 1-1

4. Gleich B, Weizenecker J (2005) Tomographic imaging using the nonlinear response of magnetic particles. Nature 435:1214-1217

5. Goodwill PW, Conolly SM (2010) The x-space formulation of the magnetic particle imaging process: 1-D signal, resolution, bandwidth, SNR, SAR, and magnetostimulation. IEEE Trans Med Imaging 29:1851-1859

6. Weizenecker J, Gleich B, Rahmer J et al (2009) Three-dimensional realtime in vivo magnetic particle imaging. Phys Med Biol 54:L1-L10

7. Neuwelt E a, Hamilton BE, Varallyay CG et al (2009) Ultrasmall superparamagnetic iron oxides (USPIOs): a future alternative magnetic resonance (MR) contrast agent for patients at risk for nephrogenic systemic fibrosis (NSF)? Kidney Int 75:465-474

8. Lu M, Cohen MH, Rieves D, Pazdur R (2010) FDA report: ferumoxytol for intravenous iron therapy in adult patients with chronic kidney disease. Am J Hematol 85:315-319

9. Spinowitz BS, Kausz AT, Baptista J et al (2008) Ferumoxytol for treating iron deficiency anemia in CKD. J Am Soc Nephrol 19:1599-1605

10. Vasanawala SS, Nguyen K-L, Hope MD et al (2016) Safety and technique of ferumoxytol administration for MRI. Magn Reson Med $75: 2107-2111$

11. Antoch G, Freudenberg LS, Beyer T et al (2004) To enhance or not to enhance? 18F-FDG and CT contrast agents in dual-modality $18 \mathrm{~F}$ FDG PET/CT. J Nucl Med 45(Suppl 1):56S-65S

12. Gleich B, Weizenecker J, Borgert J (2008) Experimental results on fast 2D-encoded magnetic particle imaging. Phys Med Biol 53:N81-N84

13. Goodwill PW, Scott GC, Stang PP, Conolly SM (2009) Narrowband magnetic particle imaging. IEEE Trans Med Imaging 28:1231-1237

14. Goodwill PW, Lu K, Zheng B, Conolly SM (2012) An x-space magnetic particle imaging scanner. Rev Sci Instrum 83:033708
15. Vogel P, Ruckert MA, Klauer $P$ et al (2014) Traveling wave magnetic particle imaging. IEEE Trans Med Imaging 33:400-407

16. Saritas EU, Goodwill PW, Croft LR et al (2013) Magnetic particle imaging (MPI) for NMR and MRI researchers. J Magn Reson 229:116-126

17. Rahmer J, Weizenecker J, Gleich B, Borgert J (2009) Signal encoding in magnetic particle imaging: properties of the system function. BMC Med Imaging 9:4

18. Goodwill PW, Conolly SM (2011) Multidimensional x-space magnetic particle imaging. IEEE Trans Med Imaging 30:1581-1590

19. Goodwill PW, Konkle JJ, Zheng B et al (2012) Projection X-space magnetic particle imaging. IEEE Trans Med Imaging 31:1076-1085

20. Konkle JJ, Goodwill PW, Hensley DW et al (2015) A convex formulation for magnetic particle imaging $\mathrm{x}$-space reconstruction. PLoS One 10:e140137

21. Ferguson RM, Khandhar AP, Kemp SJ et al (2015) Magnetic particle imaging with tailored iron oxide nanoparticle tracers. IEEE Trans Med Imaging 34:1077-1084

22. Ferguson RM, Khandhar AP, Krishnan KM (2012) Tracer design for magnetic particle imaging (invited). J Appl Phys 111:7B318-7B3185.

23. Rahmer J, Antonelli A, Sfara C et al (2013) Nanoparticle encapsulation in red blood cells enables blood-pool magnetic particle imaging hours after injection. Phys Med Biol 58:3965-3977

24. Zheng B, Vazin T, Goodwill PW et al (2015) Magnetic particle imaging tracks the long-term fate of in vivo neural cell implants with high image contrast. Sci Rep 5:14055

25. Zheng B, von See MP, Yu E et al (2016) Quantitative magnetic particle imaging monitors the transplantation, biodistribution, and clearance of stem cells in vivo. Theranostics 6:291-301

26. Vogel P, Rückert MA, Klauer P et al (2016) First in vivo traveling wave magnetic particle imaging of a beating mouse heart. Phys Med Biol 61:6620-6634

27. Saritas EU, Goodwill PW, Zhang GZ, Conolly SM (2013) Magnetostimulation limits in magnetic particle imaging. IEEE Trans Med Imaging 32:1600-1610

28. Saritas EU, Goodwill PW, Conolly SM (2015) Effects of pulse duration on magnetostimulation thresholds. Med Phys 42:3005-3012

29. Schmale I, Gleich B, Schmidt J, et al. (2013) Human PNS and SAR study in the frequency range from 24 to $162 \mathrm{kHz}$. In: 2013 International Workshop on Magnetic Particle Imaging (IWMPI). ieeexplore.ieee.org, pp 1-1

30. Borgert J, Schmidt JD, Schmale I et al (2013) Perspectives on clinical magnetic particle imaging. Biomed Tech 58:551-556

31. Yu EY, Bishop M, Zheng B et al (2017) Magnetic particle imaging: a novel in vivo imaging platform for cancer detection. Nano Lett. doi:10.1021/acs.nanolett.6b04865

32. Zhou XY, Jeffris $\mathrm{K}, \mathrm{Yu} \mathrm{E}$ et al (2017) First in vivo magnetic particle imaging of lung perfusion in rats. Phys Med Biol. doi:10.1088/1361$6560 / \mathrm{aa} 616 \mathrm{c}$

33. Lu K, Goodwill PW, Saritas EU et al (2013) Linearity and shift invariance for quantitative magnetic particle imaging. IEEE Trans Med Imaging 32:1565-1575

34. Konkle JJ, Goodwill PW, Saritas EU et al (2013) Twenty-fold acceleration of $3 \mathrm{D}$ projection reconstruction MPI. Biomed Tech 58:565-576

35. Wawrzik T, Kuhlmann C, Ludwig F, Schilling M (2013) Scanner setup and reconstruction for three-dimensional magnetic particle imaging. In: Weaver JB, Molthen RC (eds) SPIE medical imaging. International Society for Optics and Photonics, p 86721B-86721B-8

36. Buzug TM, Bringout G, Erbe $M$ et al (2012) Magnetic particle imaging: introduction to imaging and hardware realization. $\mathrm{Z}$ Med Phys 22:323-334

37. Murase K, Hiratsuka S, Song R, Takeuchi Y (2014) Development of a system for magnetic particle imaging using neodymium magnets and gradiometer. Jpn J Appl Phys 53:067001

38. Panagiotopoulos N, Duschka RL, Ahlborg M et al (2015) Magnetic particle imaging: current developments and future directions. Int $\mathrm{J}$ Nanomedicine 10:3097-3114

39. Yu E, Goodwill P, Tay ZW, et al (2016) In vivo projection imaging and $3 \mathrm{D}$ computed tomography magnetic particle imaging with a high resolution $6 \mathrm{~T} / \mathrm{m}$ field free line electromagnet. Proceedings of world molecular imaging congress 2016

40. Nguyen PK, Riegler J, Wu JC (2014) Stem cell imaging: from bench to bedside. Cell Stem Cell 14:431-444 
41. MPI - Bruker's revolutionary modality for preclinical imaging. In: Bruker Biospin. https://www.bruker.com/fileadmin/user_upload/8PDF-Docs/PreclinicalImaging/Brochures/MPI-PreClinicalBrochure.pdf. Accessed 24 Jan 2017

42. MOMENTUM ${ }^{\mathrm{TM}}$ Imager Magnetic Particle Imaging System. In: Magnetic Insight. http://www.magneticinsight.com/momentum-imager/. Accessed 24 Jan 2017

43. Schmale I, Gleich B, Kanzenbach J, et al (2009) An introduction to the hardware of magnetic particle imaging. In: World congress on medical physics and biomedical engineering, September 7-12, 2009, Munich, Germany. Springer Berlin Heidelberg, pp 450-453

44. Schmale I, Gleich B, Borgert J, Weizenecker J (2010) JFET noise modelling for MPI receivers. Proceedings of IWMPI 148-153.

45. Khandhar AP, Keselman P, Kemp SJ et al (2017) Evaluation of PEGcoated iron oxide nanoparticles as blood pool tracers for preclinical magnetic particle imaging. Nano 9:1299-1306

46. Bulte JWM, Walczak P, Janowski M et al (2015) Quantitative "hot spot" imaging of transplanted stem cells using superparamagnetic tracers and magnetic particle imaging (MPI). Tomography 1:91-97

47. Croft LR, Goodwill PW, Conolly SM (2012) Relaxation in x-space magnetic particle imaging. IEEE Trans Med Imaging 31:2335-2342
48. Croft LR, Goodwill PW, Konkle JJ et al (2016) Low drive field amplitude for improved image resolution in magnetic particle imaging. Med Phys 43:424-435

49. Rahmer J, Halkola A, Gleich B et al (2015) First experimental evidence of the feasibility of multi-color magnetic particle imaging. Phys Med Biol 60:1775-1791

50. Hensley D, Goodwill P, Croft L, Conolly S (2015) Preliminary experimental x-space color MPI. In: 2015 5th International Workshop on Magnetic Particle Imaging (IWMPI). pp 1-1

51. Hensley DW, Tay ZW, Dhavalikar R et al (2016) Combining magnetic particle imaging and magnetic fluid hyperthermia in a theranostic platform. Phys Med Biol. doi:10.1088/1361-6560/aa5601

52. Bauer LM, Situ SF, Griswold MA, Samia ACS (2016) Highperformance iron oxide nanoparticles for magnetic particle imaging — guided hyperthermia (hMPI). Nano 8:12162-12169

53. Kuboyabu T, Yamawaki M, Aoki M, et al (2016) Quantitative evaluation of tumor early response to magnetic hyperthermia combined with vascular disrupting therapy using magnetic particle imaging. Int J Nanomed Nanosurg 2. doi 10.16966/2470-3206.114

54. Keselman P, Yu E, Zhou X et al (2017) Tracking short-term biodistribution and long-term clearance of SPIO tracers in magnetic particle imaging. Phys Med Biol. doi:10.1088/1361-6560/aa5f48 